Somnologie 2011 · 15:69-69

DOI 10.1007/s11818-011-0516-y

Online publiziert: 13. Mai 2011

(c) Springer-Verlag 2011

\title{
T. Podszus
}

Abt. Pneumologie, internistische Intensivmedizin, Schlafmedizin, Medizinische Klinik, Sana-Klinikum Hof

\section{Die Entwicklung der Schlafmedizin in Deutschland}

Die Schlafmedizin in Deutschland hat in den letzten 40 Jahren einen grundlegenden Wandel von einer forschenden Wissenschaft hin zur flächendeckenden Versorgung von Patienten mit schlafmedizinischen Problemen erfahren. Standen am Anfang die Beschreibung physiologischer Abläufe und das Verständnis um den Ablauf des normalen und gestörten Schlafs im Fokus, so traten sehr bald die Probleme in den Vordergrund, die sich aus der Diagnosestellung des gestörten Schlafs und der hieraus resultierenden Notwendigkeit einer therapeutischen Intervention ergaben. Hierbei entstanden, insbesondere mit der schnell voran schreitenden Entwicklung moderner Sensortechnologien, zunehmende diagnostische Möglichkeiten im Schlaf wie auch im Wachzustand, sodass auch die Betrachtungsweise des Schlafs als Krankheitsfaktor bzw. krankheitsinduzierender Faktor neu definiert werden musste.

Folgerichtig änderten sich die diagnostischen und therapeutischen Bemühungen hin zur Behandlung von Störungen im Schlaf-Wach-System. Zunehmende theoretische und wissenschaftliche Einsicht in die Abläufe des normalen und gestörten Schlaf-Wach-Rhythmus aus dem Bereich der Biorhythmik müssen ebenfalls in die Beurteilung einbezogen werden, sodass heute die Probleme des „nichterholsamen Schlafs“ im Mittelpunkt der Schlafmedizin stehen. Diese Entwicklung bezieht sich einerseits auf die weiter fortschreitenden wissenschaftlichen Bestrebungen im Bereich der Schlafmedizin und hat aber auch Eingang in die Patientenversorgung in Form einer S3Leitlinie gefunden. Somit hat die Schlafmedizin in Deutschland sich als eine sich im permanenten geistigen Wandel befindende Wissenschaft erwiesen.

Eine weitere Besonderheit hat die Entwicklung der Schlafmedizin in Deutschland geprägt. $\mathrm{Zu}$ Beginn wurde die Forschung von Wissenschaftlern aus der Neurophysiologie betrieben. Andere medizinische Fakultäten nahmen den Schlaf als Teil von Krankheitsabläufen kaum oder gar nicht wahr. Im Verlauf entstanden vereinzelt Schlaflaboratorien zu Forschungszwecken in psychiatrischen und neurologischen Kliniken. In Deutschland gründete Professor Peter an der Universität Marburg 1981 das erste Schlaflabor in einer internistischen Klinik bzw. in einer schwerpunktmäßig pneumologischen Abteilung. Der initiale Gedanke, hier auch primär bei selektierten Patienten wissenschaftlich tätig zu sein, musste sehr schnell der Wirklichkeit Rechnung tragen, die sich aus einer zunehmenden Anzahl von Patienten ergab. So wurde durch das hohe Patientenaufkommen auch der Weg der Entwicklung diagnostischer und therapeutischer Strategien bestimmt.

Durch die Kenntnis vieler Patienten erkannte Professor Peter, dass eine ausschließlich fachbezogene Betrachtung den Problemen der Schlafmedizin nicht gerecht wird. Daraus folgte, dass interdisziplinäre Ansätze erarbeitet werden mussten, die bis heute das Bild der Schlafmedizin in Deutschland prägen. So sind neben der Neurologie und Psychiatrie auch die innere Medizin mit der Kardiologie, Pneumologie, Nephrologie und Endokrinologie, die HNO-Heilkunde, die MundKiefer-Gesichts-Chirurgie, die Orthopädie und die Pädiatrie Teil der Interdisziplinarität der Schlafmedizin. Die Notwen- digkeit dieser Entwicklung hat Professor Peter schon sehr frühzeitig erkannt und gefördert, sodass 1992 diese Entwicklung in die übergreifende „Deutsche Gesellschaft für Schlafforschung und Schlafmedizin" mündete.

Durch raschen theoretischen Fortschritt in wissenschaftlichen Erkenntnissen wie auch in den technischen Entwicklungen haben die Beurteilung von Störungen des "nichterholsamen Schlafs“ wie auch die Beurteilung vieler Krankheitsabläufe grundlegende Wandlungen erfahren. Insbesondere in der inneren Medizin führt die Betrachtung vieler Erkrankungen im Schlaf-Wach-Rhythmus zu neuen Ansätzen in Diagnostik und Therapie. Professor Peter hat bereits in den 1980erJahren auf die komplexen Zusammenhänge zwischen Herz-Kreislauf-Erkrankungen und schlafbezogenen Atmungsstörungen hingewiesen. Heute sind viele dieser Zusammenhänge anerkannter Standard für Diagnostik und Therapie.

Die Interdisziplinarität, der wissenschaftlich forschende Ansatz und die Notwendigkeit der flächendeckenden Versorgung von Patienten mit schlafmedizinischen Problemen haben die Einsicht in viele Krankheitsabläufe und das Verständnis physiologischer und pathophysiologischer Abläufe erweitert und teilweise neu definiert.

\section{Korrespondenzadresse \\ T. Podszus}

Abt. Pneumologie, internistische Intensivmedizin, Schlafmedizin, Medizinische Klinik, Sana-Klinikum Hof Eppenreuther Str. 9, 95032 Hof thomas.podszus@klinikumhof.de 